\title{
Can the anode heel effect be used to optimise radiation dose and image quality for AP pelvis radiography?
}

\begin{abstract}
$\underline{\text { Abstract }}$
Introduction:

A study was conducted to determine whether the anode heel effect can be used to influence optimisation of radiation dose and image quality (IQ) for AP pelvis radiography.

Methods:

ATOM dosimetry phantom and an anthropomorphic phantom were positioned for AP pelvis. Using a CR system, images were acquired and doses were measured with phantom feet toward anode and then feet toward cathode. Exposure factors (kVp, mAs and SID) were systematically generated using a factorial design. Images were scored visually for quality using relative visual grading together with a 3 point Likert scale. Signal to noise ratio was also calculated as a physical measure of image quality. Dosimetry data were collected for the ovaries and testes.
\end{abstract}

\section{Results:}

The optimum technique for male, which resulted in lower dose and suitable image quality, was with feet positioned toward the anode $(0.80 \pm 0.03 \mathrm{mGy}$; SNR of $38 \pm 2.9$; visual IQ score $3.13 \pm$ 0.35). The optimum technique for female was with feet toward anode $(0.23 \pm 0.02 \mathrm{mGy}$; SNR of 34.7 \pm 2.6 ; visual IQ score $3.15 \pm 0.26$ ). $\mathrm{kVp}$ had the biggest effect on both visual and physical image quality metrics $(\mathrm{p}<0.001)$ for both tube orientations, whereas SID had the lowest effect on both visual and physical image quality metrics compared with $\mathrm{mAs}$ and $\mathrm{kVp}(\mathrm{p}<0.001)$. The effect of SID on the SNR was not significant ( $p>0.05$ ) with feet toward anode.

\section{Conclusion:}

Positioning the patient with feet toward the anode, as opposed to the cathode, has no adverse effect on visual image quality assessment but it does have an effect on physical image quality.

\section{Implications for Practice:}

This study would add a new clinical concept in positioning of AP pelvis radiography especially for male positioning.

Keywords: AP pelvis, anode heel effect, optimisation, image quality, gonad dose. 


\section{Introduction}

Anteroposterior (AP) pelvis radiography is the third most frequently conducted diagnostic X-ray examination in UK. ${ }^{1,2}$ With respect to the gonads, radiation protection is of paramount importance since irradiation of germ cells has the potential to cause genetic mutations. ${ }^{3}$ A confounding factor in computed radiography (CR) is dose creep, in which a radiation dose increase can occur over time with no change in image quality, because of the wide dynamic range of CR X-ray imaging technologies. Such an increase can permit significant over-exposure without penalty to image quality. ${ }^{4,5}$ The search for an optimal approach that reduces patient dose whilst maintaining an image of diagnostic quality is a priority. ${ }^{6,7}$ The latter can be implemented by identifying strategies which minimise the occurrence of patient overexposure, thereby reducing the probability of dose creep. $^{8}$

Manipulating primary exposure factors (tube potential $(\mathrm{kVp})$ and tube load $(\mathrm{mAs})^{9-11}$, varying the distance between X-ray tube and patients' skin $^{12,13}$ and the use of an air gap are approaches that have been used to optimise radiation dose and image quality for AP pelvis radiography. ${ }^{14} \mathrm{~A}$ phantom based study to optimise the AP pelvis radiography in relation to AEC chambers orientation has been conducted. ${ }^{2}$ However, non-systematic manipulation of $\mathrm{kVp}$ and mAs may lead to a loss of image quality / radiation dose benefits. ${ }^{15}$ The impact of anode heel on X-ray beam intensity from anode to cathode has been found to differ by as much as $45 \% .{ }^{16}$ Several studies ${ }^{17-19}$ have considered the impact of the anode heel effect on image quality and one $\operatorname{study}^{20}$ has considered the orientation effect on image quality and effective dose using the two lateral AEC chambers.

To date no study has considered the effect of anode heel on image quality and gonad doses for AP pelvis with manual exposure settings. In this regard, recently we investigated the potential of using the anode heel effect to reduce AP pelvis gonad dose, however we did not investigate image quality. ${ }^{21}$ Other works ${ }^{17-20}$ which considered the effect of tube orientation on AP pelvis radiography have not considered how the combination of the anode heel effect with systematic manipulation of exposure factors would impact on gonad dose and image quality. Consequently, the research presented here revisits the gonad dose whilst simultaneously investigating whether anode-cathode orientation has an impact on image quality. 


\section{Methods}

A Wolverson Arcoma Arco Ceil (Arcoma, Annavägen, Sweden) general radiography X-ray system and high frequency generator with VARIAN $130 \mathrm{HS}$ X-ray tube with $3 \mathrm{~mm} \mathrm{Al} \mathrm{total}$ filtration was used to make all exposures. Quality control results (i.e tube output, $\mathrm{kVp}$, timer consistency, $\mathrm{kVp}$ and timer accuracy, $\mathrm{kVp}$ linearity, dose output variation with $\mathrm{mA}$, and $\mathrm{kVp}$ variation with $\mathrm{mA}$ ) fell within manufacturer limits. One $35 \times 43 \mathrm{~cm}$ AGFA CR MD 4.0 cassette (Siemens, Munich, Germany) was used for imaging in our experiments; this was processed in a $35-X$ reader (Siemens, Munich, Germany) with a spatial resolution of 10 pixels/mm and grey scale resolution of 12 bits/pixel. ${ }^{22}$ A reciprocating grid with 10:1 ratio, 40 line/cm frequency, and focused strips was used (Wolverson, Willenhall, UK). An Unfors Mult-O-Meter 401 was used to calibrate TLDs.

\section{Anthropomorphic phantom image acquisition}

An anthropomorphic phantom (Rando®, The Phantom Laboratory, and USA) was used to generate representative images of a human pelvis. It is constructed of tissue equivalent materials with an embedded natural human skeleton to provide realistic conditions for testing acquisition protocols and image processing technology. ${ }^{23,24}$ Anthropomorphic phantom images were acquired with feet towards the anode and then, in the opposite orientation, with feet towards the cathode. The phantom was positioned in accordance with the literature. ${ }^{25}$ Exposure factors were identified using a factorial design, $\mathrm{k}^{\mathrm{n}}$, where $\mathrm{n}$ represents the number of exposure factors (e.g kVp) and $\mathrm{k}$ represents the number of levels for each exposure factor. ${ }^{26}$ This resulted in 64 exposure factor settings (4×4×4), comprising: 70, 75, 80 and $85 \mathrm{kVp}$; 18, 22, 27 and $32 \mathrm{mAs} ; 105,110,115$ and $120 \mathrm{~cm}$ SID - 128 images in total for both orientations. These factors are typical of those used in clinical settings for AP pelvis imaging. ${ }^{27,28}$ The same 64 exposures were repeated for both phantom orientations (64 x 2 matched pairs).

\section{Gonad dose measurement}

The method used to record gonadal radiation dose has been published previously ${ }^{21}$, but for completeness we recap the method and data in this paper. An adult dosimetry phantom (701-B A CIRS ATOM) was used for direct dose measurements. ${ }^{29}$ Two thermoluminescent detector (TLD) pellets were placed in the region which represents the male and female gonads (Figure 1). High sensitivity LiF Mg, Cu (P-100H) TLDs were used. ${ }^{30,31}$ TLDs were grouped into batches of similar response in order to have homogenous groups. Any TLD that deviated $>1.5 \%$ SD from group mean 
was removed. Linearity of TLDs was tested over a range of doses (correlation, $\mathrm{R}^{2}=0.994$ ) and intra-class correlation was used to test TLD reliability (consistency coefficient $=0.99-1.00$ ). In accordance with manufacturer instructions, TLDs were annealed at $240^{\circ} \mathrm{C}$ for 10 minutes and then cooled to room temperature to remove any residual dose from previous exposures.

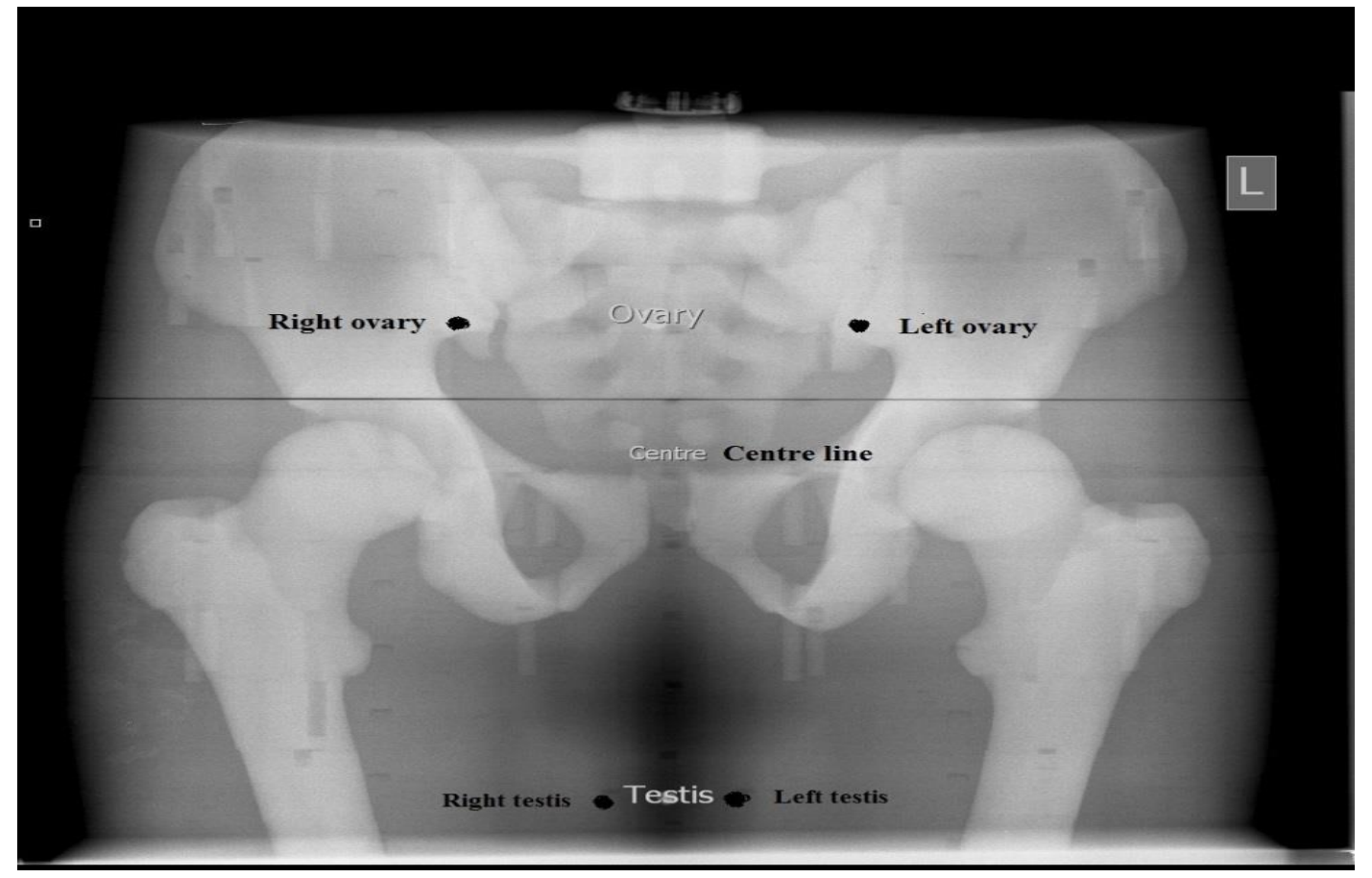

Figure 1. Locations of the ovaries and testes where TLD's were placed within the ATOM phantom.

\section{Image quality assessment}

Images were assessed visually using relative visual grading with images being displayed within bespoke image display software. ${ }^{32}$ Two reporting grade 5 mega pixel monochrome liquid crystal (LCD) monitors were used to display the images; these were calibrated to the DICOM Greyscale standard and located in ambient lighting at below 8 lux. ${ }^{33}$ The reference image was chosen on the basis of the median SNR value recorded from the 128 images (64 in each orientation). ${ }^{11}$ This reference image was displayed on one monitor whilst the images to be evaluated were displayed in a random order on the other monitor. Nevertheless, the suitability of the quality of the reference image was further checked by testing the adequate visualisation of scale criteria using a visual grading approach, hence all items (Table 1) needed to be adequately visualised (have a score of 3 or more for each criterion) in order for that image to be selected as the reference image ${ }^{34}$, so that 
any of the optimised (experimental) images that scored as equal to or better than the reference image could be considered as an image with acceptable quality

\begin{tabular}{|c|l|}
\hline \multicolumn{2}{|c|}{ Table1. AP pelvis scale for image quality assessment. } \\
\hline Item No & \\
\hline 1 & The left hip joint is visualised. \\
\hline 2 & The right hip joint is visualised. \\
\hline 3 & The right lesser trochanter is visualised. \\
\hline 4 & The left lesser trochanter is visualised. \\
\hline 5 & The left greater trochanter is visualised. \\
\hline 6 & The right greater trochanter is visualised. \\
\hline 7 & The left iliac crest is visualised. \\
\hline 8 & The right iliac crest is visualised. \\
\hline 9 & The pubic and ischial rami are visualised. \\
\hline 10 & The proximal femora are demonstrated. \\
\hline 11 & The left femoral neck is visualised. \\
\hline 12 & The right femoral neck is visualised. \\
\hline 13 & The left sacro-iliac joint is visualised. \\
\hline 14 & The right sacro-iliac joint is visualised. \\
\hline 15 & The sacrum and its intervertebral foramina are visualised. \\
\hline
\end{tabular}

Visual image quality criteria (Table 1) were used ${ }^{34}$ along with a 3 point Likert scale (score of 2 through 3 to 4), to allow grading from 'worse-than' through 'same' to 'better-than'. One experienced observer (a medical physicist with a background in radiography $>5$ years' experience) scored the images. Each image took around 60 seconds to be assessed (i.e. 4 seconds for each criterion). After assessing the first set of 64 images, a break was allowed (30 minutes) to reduce the impact of fatigue. Prior to performing visual grading, this observer was assessed against three experienced radiographers with $>10$ years' experience to determine his ability. Twenty images were selected, based on SNR values, with qualities including poor, average and good. Imaging scoring occurred three times, once per week for 3 weeks. Interclass Correlation Coefficient (ICC) was calculated to assess intra and inter observer variability. Finally, signal to noise ratio (SNR) was calculated as a physical metric of image quality and Figure 2 indicates where the regions of interest were placed. ROIs were positioned manually on the first image and for subsequent images they were positioned automatically using Image J software. The SNR was calculated to support the visual assessment of image quality. 


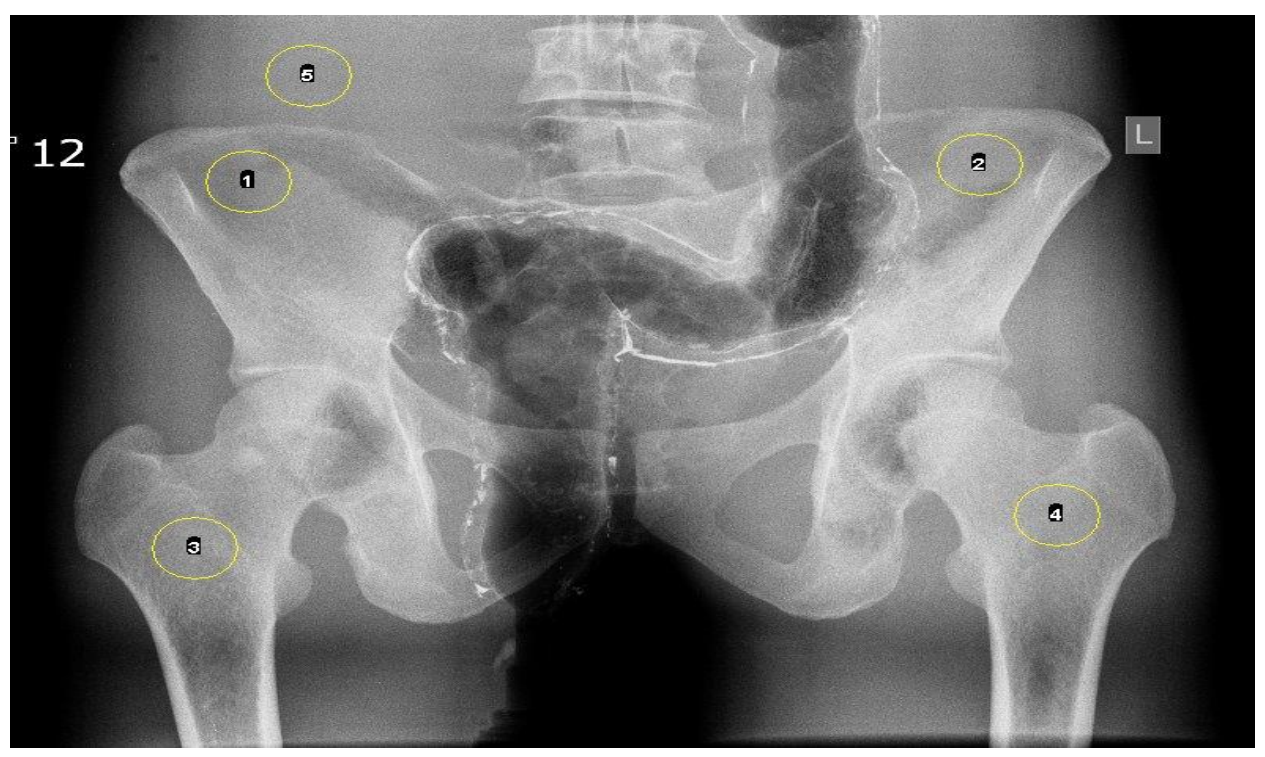

Figure 2: Regions of interest used for SNR calculations with optimised images taken using the Anthropomorphic pelvis phantom (bone=1-4, which were averaged; background=5).

\section{Results}

The interclass correlation coefficient for study observer when compared to the three radiographers was 0.839 (95\% CI 0.683 to 0.929 ) - an ICC value of less than 0.40 indicates poor reproducibility whereas ICC values greater than 0.75 shows an excellent reproducibility. ${ }^{2}$ The resultant ICC values for intra-observer variability were, 0.891 (95\%CI 0.725 to 0.957 ), 0.920 (95\%CI 0.798 to 0.968 ) and 0.889 (95\%CI 0.725 to 0.956 , respectively). This confirmed the observer (medical physicist) was able to produce valid scores. Image quality scores were assessed using t-tests with a p-value of $<0.05(95 \%)$ being considered as significant.

In general, the resultant data demonstrated that the anode heel effect had an impact on the male gonad dose for both orientations with no effect on female gonad dose. No effect was found when considering the anode heel effect on the visual image quality and for either orientation; there was an effect for SNR between the two orientations.

Exposure factors which led to images of acceptable quality (optimised) using relative visual grading, which had the lowest testicular dose when feet were faced toward the anode, were identified $(0.80 \mathrm{mGy})$. Significant differences $(\mathrm{P}<0.05)$ were found in the testicular doses between both orientations. An acceptable quality image (optimised) was acquired when the head was placed toward the cathode at a testicular dose of $0.99 \mathrm{mGy}$. No significant differences $(\mathrm{P}>0.05)$ were found in the ovarian dose between either orientations of the phantom relative to the anode; the 
lowest dose with acceptable quality image (optimised) was obtained when head was placed toward the cathode $(0.22 \mathrm{mGy})$. When feet faced the anode, the lowest female gonad dose for achieving an acceptable image quality was $0.23 \mathrm{mGy}$. The optimal exposure factors which led to an acceptable image quality (male \& female), with the corresponding physical metrics, are presented in table 2. No significant difference was detected in terms of relative visual grading image quality scores between the two anode orientations $(\mathrm{P}>0.05$; feet toward anode: $3.16 \pm 0.46 \mathrm{Vs}$ feet toward cathode: $3.26 \pm 0.43)$.

\begin{tabular}{|c|c|c|c|c|c|c|}
\hline \multirow{2}{*}{ Orientation } & \multirow{2}{*}{ Organs } & \multicolumn{3}{|c|}{ Optimum exposure Factors } & \multirow{2}{*}{ Dose (SD) } & \multirow{2}{*}{ SNR (SD) } \\
\hline & & $\mathbf{k V p}$ & mAs & SID & & \\
\hline \multirow{2}{*}{$\begin{array}{c}\text { Feet toward } \\
\text { cathode }\end{array}$} & Testes & 80 & 22 & 120 & $0.99(0.03)$ & $34.0(2.0)$ \\
\hline & Ovaries & 75 & 22 & 115 & $0.22(0.02)$ & $34.0(1.0)$ \\
\hline \multirow{3}{*}{$\begin{array}{c}\text { Reference } \\
\text { image data } \\
\text { (Toward } \\
\text { Cathode) }\end{array}$} & Testes & \multirow{3}{*}{75} & \multirow{3}{*}{18} & \multirow{3}{*}{105} & $1.38(0.01)$ & \multirow{3}{*}{$24.7(3.6)$} \\
\hline & & & & & & \\
\hline & & & & & & \\
\hline \multirow{2}{*}{$\begin{array}{c}\text { Feet toward } \\
\text { anode }\end{array}$} & Testes & 80 & 18 & 110 & $0.80(0.03)$ & $38.0(2.9)$ \\
\hline & Ovaries & 70 & 28 & 115 & $0.23(0.02)$ & $34.7(2.6)$ \\
\hline \multirow{2}{*}{$\begin{array}{c}\text { Reference } \\
\text { image data } \\
\text { (Toward } \\
\text { Anode) }\end{array}$} & Testes & \multirow[b]{2}{*}{70} & \multirow[b]{2}{*}{28} & \multirow[b]{2}{*}{105} & $1.14(0.05)$ & \multirow[b]{2}{*}{$25.7(2.31)$} \\
\hline & Ovaries & & & & $0.35(0.01)$ & \\
\hline SD. St & ion & & & & & \\
\hline
\end{tabular}

When comparing the mean SNR for all images between feet toward anode and feet toward cathode, there was a statistical difference between the two orientations $(\mathrm{P}<0.05$; feet toward anode: $37.90 \pm 4.57$ Vs. feet toward cathode: $35.71 \pm 5.09$ ).

The main effect of the exposure factors on visual image quality scores is illustrated in Figure 3 (A and B) for both anode orientations. Increasing the $\mathrm{kVp}$ had the biggest effect (Significant at 
$\mathrm{p}<0.001$ ) on relative visual grading image quality since it resulted in an increase in visual image quality by approximately $24 \%$ with feet toward cathode and $31 \%$ with feet toward anode, respectively. Increasing the mAs from 18 to 32 resulted in an increase in visual image quality by approximately 19\% with feet toward cathode and $23 \%$ with feet toward anode, respectively (Significant effect at $\mathrm{p}<0.001$ ). SID had the smallest effect on visual image quality scores. Increasing the SID resulted in a decline in visual image quality by approximately $7 \%$ with feet toward cathode and $9 \%$ with feet toward anode, respectively (Significant effect at $\mathrm{p}<0.001$ ).
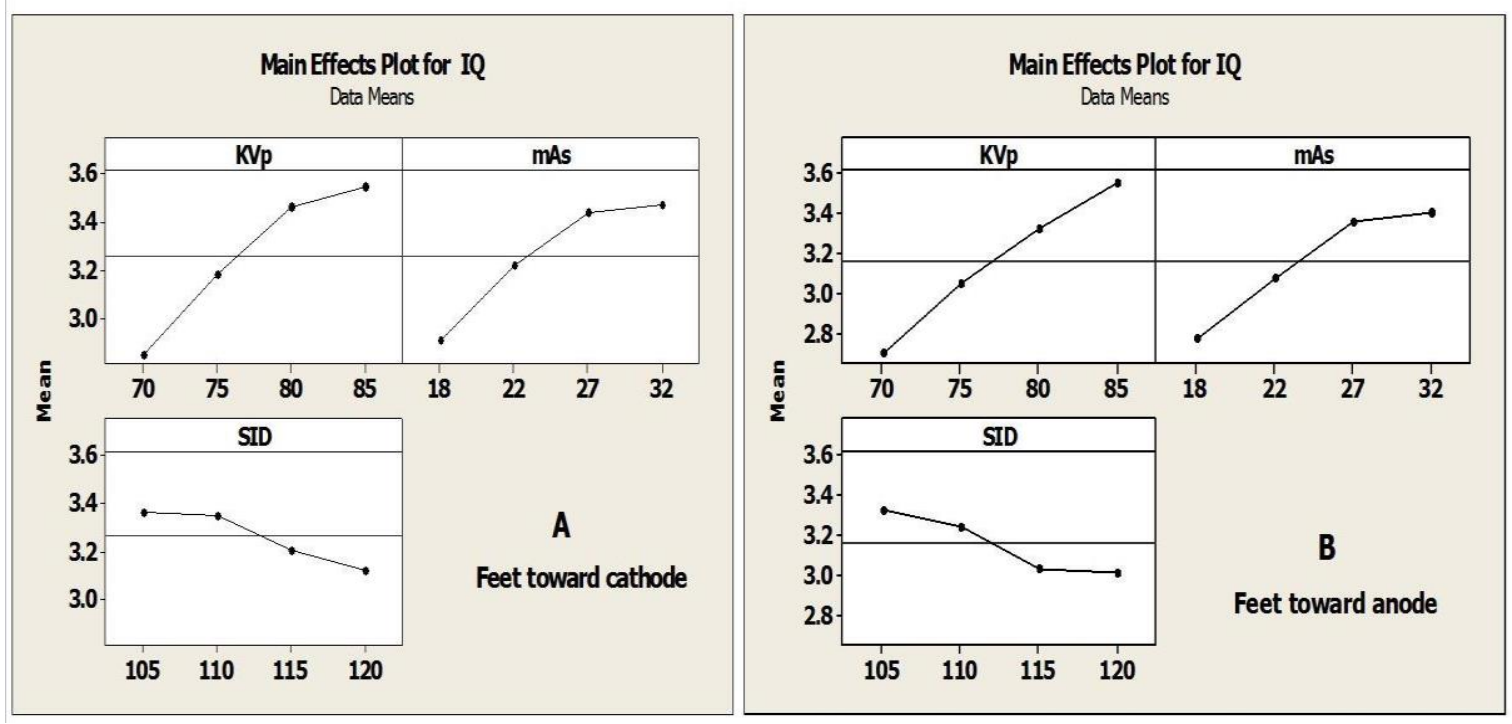

Figure 3. This figure illustrates the main effect of exposure factors on the mean of visual Image Quality (IQ) score when the exposure factors were varied (from low to high) for both tube orientations(A and B). The horizontal lines represent the image quality mean (overall mean of all images).

The main effect of the exposure factors on SNR is illustrated in Figure 4 (A and B) for both anode orientations. As it can be seen, the mAs had the biggest effect on the SNR since it resulted in an increase in SNR by approximately 20\% with feet toward cathode and 19.5\% with feet toward anode, respectively (Significant effect at $\mathrm{p}<0.001$ ). Increasing the $\mathrm{kVp}$ resulted in an increase in SNR by approximately $17 \%$ for both tube orientations (Significant effect at $p<0.001$ ). The SID had approximately the smallest effect on SNR for both orientations. 

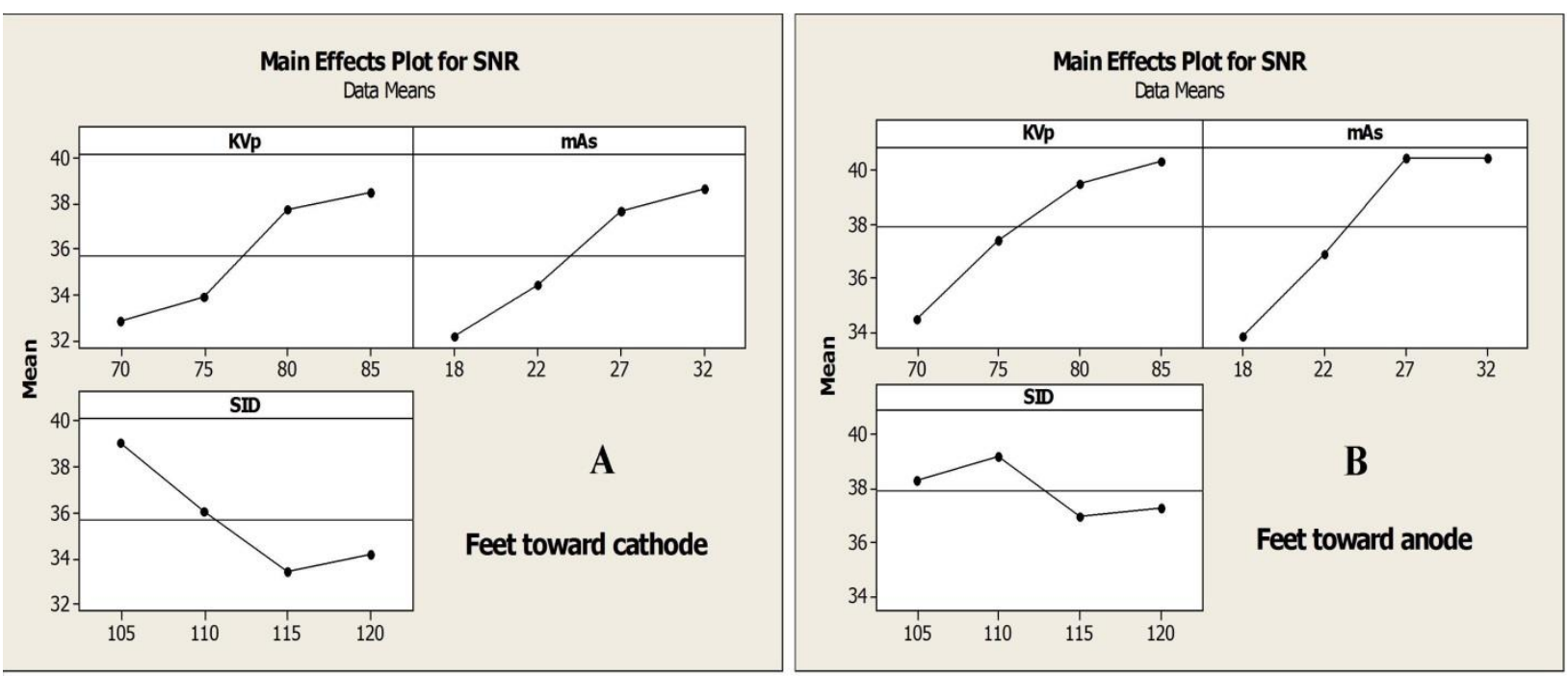

Figure 4. This figure illustrates the main effect of exposure factors on the signal to noise ratio (SNR) when the exposure factors were varied (from low to high) for both tube orientations. The horizontal lines represent the image quality mean (overall mean of all images).

A good correlation was seen between SNR and relative visual grading image quality scores for both tube orientations $(\mathrm{r}=0.853$; significant at $\mathrm{p}<0.001)$. As previously confirmed ${ }^{16}$, there is a statistically significant difference between the two orientations for testicular dose $(\mathrm{P}<0.001)$ but there is no significant difference for ovarian dose ${ }^{21}$.

\section{Discussion}

Given that no statistical difference was found in terms of relative visual grading image quality scores between the two orientations, this finding offers radiographers the opportunity to place the patient's feet toward anode with no image quality consequences whilst having a marked reduction in the male gonadal dose. The significant difference in SNR values, between the two orientations, favours feet toward the anode. Positioning the feet towards anode was also beneficial for females in terms of image quality, however there was no radiation dose reduction by doing this.

In relation to image quality, both anode heel orientations were associated with a range of images of suitable quality. However, for males, the optimum orientation that led to the lowest radiation dose with suitable image quality was with feet towards the anode. By contrast, both orientations can be adopted for females, since no radiation dose implications were seen. This means that switching phantom orientation would not affect the quality of an image. Therefore, the anode heel 
effect can provide a good margin for protecting the male patient from unnecessary radiation consequences. In addition, placing the thinner parts of the pelvis toward the anode and thicker parts toward the cathode comply with published recommendations regarding using the heel effect as a wedge filter. ${ }^{35,36}$

However, the results of our study contradict Al Qaroot et al (2014) ${ }^{19}$, who investigated the anode heel effect on radiation dose and image quality for lateral lumbar spine. The authors recommend positioning the feet toward cathode, depending on the number of suitable quality images acquired with a low effective dose in relation to cathode-anode orientation. Optimising the anode heel effect using effective dose would ignore the impact of the X-ray intensity variation on organ doses, and therefore on radiation risk estimation. ${ }^{37}$ This is because estimating effective dose is always based on the measured incident air kerma at the centre of the X-ray beam, where the intensity is $100 \%$ compared with either side. This therefore makes comparison difficult in terms of radiation dose since no gonad dose measurements were considered in this study. Although Harding et al ${ }^{20}$ findings are consistent with our findings, again it is difficult to make a comparison since their work does not consider the heel effect on the beam intensity as it actually occurs.

Another contradiction was observed between the findings of our work and Mearon \& Brennan $(2006)^{17}$, who studied the possibility of improving image quality during thoracic spine examination in relation to anode orientation. Consequently, they recommend placing the feet towards the cathode rather than the anode, because this slightly improves image quality by exposing the thicker body parts to a higher X-ray intensity, compared with the thinner body parts. However, it is difficult to compare this with the recommendations drawn from our study for two reasons: first, their study was conducted using analogue and not digital systems; secondly they did not consider the anode heel effect on radiation dose.

The main effect on image quality, Figure 3 (A and B), demonstrates that $\mathrm{kVp}$ had the largest effect on image quality for both anode orientations, compared with the mAs and SID. The effect trends in relation to both $\mathrm{kVp}$ and mAs levels increase proportionally before levelling off at $80 \mathrm{kVp}$ and $27 \mathrm{mAs}$. Image quality improvement as the $\mathrm{kVp}$ increases from 70 to 85 is not controversial since, with high $\mathrm{kVp}$ more photons reach the detector; the amount of noise is reduced. Nevertheless, at very high $\mathrm{kVp}$ 's, subject contrast reduces due to the low attenuation characteristics of the tissues being imaged. ${ }^{38,39}$ SID had the smallest effect on image quality. 
The effect that SID had on reducing dose is larger than the impact on the image quality, when distance increased. This provides the opportunity to use SID as a method of optimisation. These findings are supported by previous published works ${ }^{12,13}$; they also found that increasing SID did not adversely affect image quality. Nevertheless, caution should be taken when using the SID in the optimisation process of any practice since grid cut off might have a detrimental impact.

Regarding the main effect on the SNR, the biggest effect of the mAs as compared to the $\mathrm{kVp}$ main effect could be attributed to the sensitivity of SNR compared with the visual measure of image quality. Altering mAs affects the number of photons reaching the image receptor and this influences the noise levels for both orientations and consequently the SNR. ${ }^{40}$ This image quality change is therefore detected using a physical measure, whereas it is not perceived using a visual measure. For the evaluation of clinical image quality, this raises the question on what traditional physical image quality measures, such as SNR, should have in to optimising [clinical] image quality as diagnostic interpretation is a visual task. Perhaps the use of Artificial Intelligence could be a way forwards in image quality optimisation, as such systems could be trained to consider critical image characteristics from both visual and physical points of view?

Finally, one limitation could concern visual image quality assessment being conducted by one observer. Whilst this might seem to be a limitation, the ICC values uphold the approach taken and obtaining several observers to score [128] images can be difficult to achieve. Also, even though our work used validated visual grading criteria, our criteria are different to those used by other researcher making results slightly difficult to compare. A need for standardised and validated visual criteria grading criteria exists.

\section{Conclusion}

The findings of our study demonstrate the anode heel effect can be used as an effective approach for optimising the AP pelvis radiographic examination for male patients using manual technique. The latter argument can be supported by the fact that positioning the [male] feet toward anode can reduce gonad dose whilst having no impact on visual image quality. The results also demonstrate, for females, that gonad dose and visual image quality are the same in either orientation. Further studies are recommended to consider the clinical implications of our work. 


\section{References}

1. Hart D, Wall BF, Hillier MC, Shrimpton PC. Frequency and collective dose for medical and dental X-ray examinations in the UK. Chilton: Health Protection Agency 2010 Contract No.: HPA-CRCE-012.

2. Manning-Stanley AS, Ward AJ, England A. Options for radiation dose optimisation in pelvic digital radiography: A phantom study. Radiography. 2012;18(4):256-63.

3. Warlow T, Walker-Birch P, Cosson P. Gonad shielding in paediatric pelvic radiography: Effectiveness and practice. Radiography. 2014;20(3):178-82.

4. Schaefer-Prokop C, Neitzel U. Computed Radiography/Digital Radiography: Radiologist Perspective on Controlling Dose and Study Quality In: Frush. DP, Huda W, editors. RSNA Categorical Course in Diagnostic Radiology Physics: From Invisible to Visible-The Science and Practice of X-ray Imaging and Radiation Dose Optimization. USA: RSNA; 2006. RSNA.

5. Gibson DJ, Davidson RA. Exposure Creep in Computed Radiography: A Longitudinal Study. Academic Radiology. 2012;19(4):458-62.

6. ICRP. The 2007 Recommendations of the International Commission on Radiological Protection.ICRP Publication 103. Annals of ICRP. 2007;37(2-4):1-332.

7. Warren-Forward HM, Beckhaus R. A standardised approach to optimisation. Radiographer. 2004 Dec;51(3):105-10.

8. Murray M. IR (ME) R 2000 and IR (ME) amendment regulations 2006 \& 2011. Society and College of Radiographers Policy and Guidance. 2012.

9. Aldrich J, Duran E, Dunlop P, Mayo J. Optimization of Dose and Image Quality for Computed Radiography and Digital Radiography. Journal of Digital Imaging. 2006;19(2):126-31.

10. Fauber TL, Cohen T, Dem psey MC. High Kilovoltage Digital Exposure Techniques and Patient Dosimetry. Radiologic technology. 2011;82(6):501-10.

11. Lança L, Franco L, Ahmed A, Harderwijk M, Marti C, Nasir S, et al. 10 kVp rule e An anthropomorphic pelvis phantom imaging study using a CR system: Impact on image quality and effective dose using AEC and manual mode. Radiography. 2014;20(4): 333-8.

12. Heath R, England A, Ward A, Charnock P, Ward M, Evans P, et al. Digital Pelvic Radiography: Increasing Distance to Reduce Dose. Raediologic technology. 2011;83(1):20-8.

13. Tugwell J, Everton C, Kingma A, Oomkens DM, Pereira GA, Pimentinha DB, et al. Increasing source to image distance for AP pelvis imaging e Impact on radiation dose and image quality. radiography. 2014;20(4): 351-5.

14. Chan CTP, Fung KLL. Dose optimization in pelvic radiography by air gap method on CR and DR systems e A phantom study. Radiography. 2015;21(3):214-23.

15. Matthews K, Brennan P. Optimisation of X-ray examinations:General and an Irish perspective. radiography. 2009;15(3):262-8.

16. Bushong SC. Radiologic science for technologists physics, biology, and protection. 3 ed. Missouri: Mosby; 2013.

17. Mearon T, Brennan PC, editors. Anode heel affect in thoracic radiology: a visual grading analysis. Medical Imaging 2006: Physics of Medical Imaging; 2006; San Diego, CA.: SPIE; 2006. 18. Soares, J. F. D. S., Dores, R. E. S., Sousa, P., Rodrigues, S., Ribeiro, L. P. V., Abrantes, A. F., \& Almeida, R. P. P. A. (2013). Attenuation of anode heel effect with an aluminum filter and their influence on patient dose in lumbar spine radiography. Paper presented at the ECR 2013, Vienna. 19. Al Qaroot, B., Hogg, P., Twiste, M., \& Howard, D. (2014). A systematic procedure to optimise dose and image quality for the measurement of inter-vertebral angles from lateral spinal 
projections using Cobb and superimposition methods. Journal of X-Ray Science and Technology, 22(5), 613-625.

20. Harding, L., Manning-Stanley, A.S., Evans, P., Taylor, E. M., Charnock, P., \& England, A. (2014). Optimum patient orientation for pelvic and hip radiography: A randomised trial. radiography, 20(1), 22-32.

21. Mraity H, Hogg P, England A. Gonad dose in AP pelvis radiography: Impact of anode heel orientation. radiography. 2017;23(1):14-8.

22. HealthCare A. CR 35-X Digitizer specifications sheet. In A. HealthCare (Ed.). 2009.

23. Inoue K, Sato T, Kitamura H, Hirayama A, Kurosawa H, Tanaka T, et al. An anthropomorphic pelvis phantom for optimization of the diagnosis of lymph node metastases in the pelvis. Annals of Nuclear Medicine. 2009;23(3):245-55.

24. Phantoms S. The Phantom Laboratory. Sectional lower Torso SK250 2014 [cited 2014 May, 1st]. Available from: http://www.phantomlab.com/library/pdf/sectional SK250DS.pdf.

25. Whitley AS, Sloane C, Hoadley G, Moore AD, Alsop CW. Clark's positioning in radiography. 12 ed. London: Hodder Arnold.; 2005

26. Montgomery DC. Design and Analysis of Experiments. USA: John Wiley \& Sons; 2013.

27. Carver B, Carver B. Medical Imaging Techniques, Reflection \& Evaluation. 2 ed. Edinburgh: Churchill Livingstone; 2012.

28. Bontrager KL, Lampignano JP. Textbook of radiographic positioning and related anatomy. 8 ed. Missouri: Mosby; 2014.

29. CIRS. ATOM dosimetry phantom :Adult male phantom model number 701-D appendix 5. Virginia, USA: CIRS, Inc2013

30. Rivera T. Thermoluminescence in medical dosimetry. Applied RadiationandIsotopes. 2012;71(Supplement):30-4.

31. Tootell AK, Szczepura KR, Hogg P. Optimising the number of thermoluminescent dosimeters required for the measurement of effective dose for computed tomography attenuation correction data in SPECT/CT myocardial perfusion imaging. Radiography. 2013;19(1):42-7.

32. Hogg, P., \& Blindell, P Software for image quality evaluation using a forced choice method. Paper presented at the UKRC, Manchster, UK; 2012.

33. Norweck JT, Seibert JA, Andriole KP, Clunie DA, Curran BH, Flynn MJ, et al. ACR-AAPMSIIM Technical Standard for Electronic Practice of Medical Imaging. Journal of digital imaging. 2013;26(1):38-52.

34. Mraity H, England A, S. C, Eachus P, A. D, Hogg P. Development and validation of a visual grading scale for assessing image quality of AP pelvis radiographic images. British journal of radiology. 2016;89(1061):1-10.

35. Katz MC, Nickoloff EL. Radiographic detail and variation of the nominal focal spot size: the "focal effect". radiographics. 1993;12(4):753-61.

36. Carlton RR, Adler AM. Principles of Radiographic Imaging: An Art and A Science 5ed. Australia: Cengage Learning; 2013.

37. Huda W. Review of radiologic physics. 3 ed. Philadelphia,: Lippincott Williams \& Wilkins; 2010.

38. Jessen KA. Balancing image quality and dose in diagnostic radiology. European Radiology Supplements. 2004;14(1):9-18.

39. Fauber TL. Radiographic imaging \& exposure. 4 ed. Missouri: Mosby; 2014.

40. Martin CJ. The importance of radiation quality for optimisation in radiology. Biomedical Imaging and Intervention Journal Commentray. 2007;3(2):1-38. 
